\title{
Asymptotic zero distribution of sections and tails of Mittag-Leffler functions
}

\author{
Natalya Zheltukhina \\ Department of Mathematics, Bilkent University, 06533 Bilkent, Ankara, Turkey \\ Received 6 May 2002; accepted 13 May 2002 \\ Note presented by Jean-Pierre Kahane.
}

\begin{abstract}
We study the asymptotic (as $n \rightarrow \infty)$ zéro distribution of $(1-\lambda) s_{n}(z)-\lambda t_{n+1}(z)$, where $\lambda \in \mathbb{C}, s_{n}$ is $n$th section, $t_{n}$ is $n$th tail of the power series of Mittag-Leffler function $E_{1 / \rho}$ of order $\rho>1$. Our results generalize the results by Edrei, Saff and Varga for the case $\lambda=0$. To cite this article: N. Zheltukhina, C. R. Acad. Sci. Paris, Ser. I 335 (2002) 133-138. (C) 2002 Académie des sciences/Éditions scientifiques et médicales Elsevier SAS
\end{abstract}

\section{Distribution asymptotique des zéros pour les sections et les restes des fonctions de Mittag-Leffler}

Résumé On étudie la distribution asymptotique (quand $n \rightarrow \infty)$ des zéros de $(1-\lambda) s_{n}(z)-$ $\lambda t_{n+1}(z)$, où $\lambda \in \mathbb{C}, s_{n}$ est la $n$ ème section, $t_{n}$ est le $n$ ème reste du developpement de la fonction de Mittag-Leffler $E_{1 / \rho}$ d'ordre $\rho>1$. On généralise les résultats obtenus par Edrei, Saff et Varga dans le cas $\lambda=0$. Pour citer cet article: $N$. Zheltukhina, C. R. Acad. Sci. Paris, Ser. I 335 (2002) 133-138. @ 2002 Académie des sciences/Éditions scientifiques et médicales Elsevier SAS

\section{Version française abrégée}

Étant donné une fonction entière transcendente (1.1), on definie par (1.2) ses sections $s_{n}(z, f)$ et ses restes $t_{n}(z, f)$. On note $R_{1}, R_{2}, R_{3}, \ldots, \lim _{n \rightarrow \infty} R_{n}=\infty$, les points de discontinuité de l'indice central de (1.1).

Soit $\mathcal{M}_{n}(\lambda, f), \lambda \in \mathbb{C}$, l'ensemble des zéros de l'équation $I_{n}\left(R_{n} z ; \lambda, f\right)=0$, où $I_{n}\left(R_{n} z ; \lambda, f\right)$ est defini (1.3). En particulier, $\mathcal{M}_{n}(0, f)$ (resp. $\mathcal{M}_{n-1}(1, f)$ ) coincide avec l'ensemble des zéros de $s_{n}\left(R_{n} z, f\right)$ $\left(t_{n}\left(R_{n-1} z, f\right)\right)$. Soit $\mathcal{M}(\lambda, f)$ l'ensemble des points d'accumulation de $\bigcup_{n=1}^{\infty} \mathcal{M}_{n}(\lambda, f)$.

En 1924, Szegö [10] a etudié l'asymptotique (quand $n \rightarrow \infty)$ de distribution des zéros de $I_{n}\left(n z ; \lambda, \mathrm{e}^{z}\right)$ pour $\lambda \in \mathbb{C}$ arbitraire. Notons, que $R_{n}=n$ pour $f(z)=\mathrm{e}^{z}$. Szegö a découvert que l'ensemble de tous les zéros de $I_{n}\left(n z ; \lambda, \mathrm{e}^{z}\right)$ est fortement lié à la courbe $S=\left\{z:\left|z \mathrm{e}^{1-z}\right|=1\right\}$ dite la courbe de Szegö. Ce lien est donné par le théorème suivant.

ThÉORÈME $S$ ([10]). - On a les égalités : (i) $\mathcal{M}\left(0, \mathrm{e}^{z}\right)=S \cap\{z:|z| \leqslant 1\}$, (ii) $\mathcal{M}\left(1, \mathrm{e}^{z}\right)=S \cap\{z$ : $|z| \geqslant 1\}$, (iii) $\mathcal{M}\left(\lambda, \mathrm{e}^{z}\right)=S$, pour $\lambda \neq 0,1$.

Dieudonné [3] a redécouvert les résultats de [10] en 1935 en utilisant une méthode différente. Les travaux de Szegö's et Dieudonné's [10], [3] ont suscité un grand intérêt aux distributions des zéros des sections et

E-mail address: natalya@ fen.bilkent.edu.tr (N. Zheltukhina). 
des restes des fonctions entières (e.g., [6] et les références de loc.cit.). En particulier, les domaines sans zéros des sections et des restes de e $\mathrm{e}^{z}$ ont été etudié dans [1,2].

En 1983, Edrei, Saff et Varga [5] ont etudié la distribution asymptotiques des zéros des sections de la fonction de Mittag-Leffler (1.4) d'ordre $\rho>1$. À la différence de la fonction exponentielle $\mathrm{e}^{z}$, la fonction de Mittag-Leffler $E_{1 / \rho}(z), \rho>1$, admet des zéros, qui donne lieu a une partie des zéros de $s_{n}\left(R_{n} z, E_{1 / \rho}\right)$ par le théorème de Hurwitz. Dans [5], il a été démontré que $s_{n}\left(R_{n} z, E_{1 / \rho}\right)$ accumulent près de la courbe $S_{1}(\rho)=S^{\prime}(\rho) \cup S^{\prime \prime}(\rho)$, où les courbes $S^{\prime}(\rho)$ et $S^{\prime \prime}(\rho)$ sont définies par (1.5) et (1.6) et appartiennent au disque unité. Plus précisement, on a le résultat suivant.

THÉORÈME ESV ([5]). $-\mathcal{M}_{0}\left(0, E_{1 / \rho}\right)=S_{1}(\rho)$, où $\mathcal{M}_{0}\left(\lambda, E_{1 / \rho}\right)$ est defini par $(1.7)$.

Ce théorème est une consequence des résultats beaucoup plus precis et compliques de [5], qui sont liés à la description des domaines sans zéros de $s_{n}\left(R_{n} z, E_{1 / \rho}\right)$. La similitude entre $E_{1 / \rho}(z)$ (donné par le Théorème ESV) et $\mathrm{e}^{z}$ (donné par Théorème $\mathrm{S}(\mathrm{i})$ ) suggère la question suivante. Est-ce que l'analogue du résultat de Szegö pour $I_{n}\left(n z ; \lambda, \mathrm{e}^{z}\right)$ est vrai pour $I_{n}\left(R_{n} z ; \lambda, E_{1 / \rho}\right)$ ? Quel est l'analogue de la partie de la courbe de Szegö $S$ qui se trouve à l'exterieur du disque unité ? Comment decrire les domaines sans zéros pour $I_{n}\left(R_{n} z ; \lambda, E_{1 / \rho}\right)$ et $\lambda \in \mathbb{C}$ arbitraire? Pour $I_{n}\left(R_{n} z ; \lambda, E_{1 / \rho}\right)$ quels sont des analogues d'autres résultats de [5] liés aux propriétés asymptotiques de $s_{n}\left(R_{n} z, E_{1 / \rho}\right)=I_{n}\left(R_{n} z ; 0, E_{1 / \rho}\right)$ ? Notre objectif dans ce travail est de répondré à ces questions.

Notre premier résultat est l'analogue direct du Théorème $\mathrm{S}$ de Szegö. On definit la corbe $S_{2}(\rho)$ par (2.1) et on pose $S(\rho)=S_{1}(\rho) \cup S_{2}(\rho)$.

THÉORÈME 1. - On a les egalités suivant : (i) $\mathcal{M}_{0}\left(0, E_{1 / \rho}\right)=S_{1}(\rho)$, (ii) $\mathcal{M}_{0}\left(1, E_{1 / \rho}\right)=S_{2}(\rho)$, (iii) $\mathcal{M}_{0}\left(\lambda, E_{1 / \rho}\right)=S(\rho)$, pour $\lambda \neq 0,1$.

Le Théorème 1 repond à la question de prolongation de la courbe $S_{1}(\rho)$ à l'exterieur du disque unité. Il implique aussi que tous les zéros de $I_{n}\left(R_{n} z ; \lambda, E_{1 / \rho}\right)$ se trouve a l'aproximité de la courbe $S(\rho)$ et de deux rayon $\arg z= \pm \pi /(2 \rho)$. Notons que la courbe $S(\rho)$ admet des asymptotes $\arg z= \pm \pi /(2 \rho)$ (tandis que la courbe de Szegö $S$ n'a pas d'asymptotes).

Le théorème suivant est concerné par des domaines sans zéros de $I_{n}\left(R_{n} z ; \lambda, E_{1 / \rho}\right)$. Il a été démontré dans [5] dans le cas $\lambda=0$ ([5], Théorème 5).

THÉORÈME 2.-Soient $\delta_{1}, \delta_{2}$ et $h$ des constantes positives. Alors, pour tout $n$ suffisament grand, $I_{n}\left(R_{n} z ; \lambda, E_{1 / \rho}\right)$ n'annule pas dans $\bigcup_{i=1}^{5} \Omega_{i}$, où les domaines $\Omega_{i}, i=1, \ldots, 5$, sont definies par (2.2).

Les deux théoremès suivants donnent des informations sur la distribution des zéros de $I_{n}\left(R_{n} z ; \lambda, E_{1 / \rho}\right)$ au voisinage des points de la courbe $S(\rho)$.

THÉORÈME 3. - Quand $n \rightarrow \infty$, (2.3) est verifié uniformement sur tout ensemble compact du plan $\zeta$.

THÉORÈme 4. - I. Soit $\xi=\xi(\phi), 0<\phi<\pi /(2 \rho)$ un point fixe sur $S^{\prime}(\rho) \cup S_{2}(\rho)$. Soit $\tau=$ $|\zeta|^{\lambda} \sin (\phi \rho)-\rho \phi$. On definit les suites $\left\{\tau_{n}\right\}_{n=1}^{\infty}$ et $\left\{\varepsilon_{n}\right\}_{n=1}^{\infty}$ par (2.4). Alors, quand $n \rightarrow \infty$, (2.5) est verifié uniformement sur tout ensemble compact du plan $\zeta$.

II. Soit $\xi=\mathrm{e}^{-1 / \rho} \mathrm{e}^{\mathrm{i} \phi}, \pi /(2 \rho)<\phi \leqslant \pi$, un point fixe sur la portion circulaire $S^{\prime \prime}(\rho)$ de $S(\rho)$. On definit les suites $\left\{\tau_{n}^{\prime}\right\}_{n=1}^{\infty}$ et $\left\{\varepsilon_{n}^{\prime}\right\}_{n=1}^{\infty}$ par (2.6). Alors, quand $n \rightarrow \infty$, (2.7) est verifié uniformement sur tout ensemble compact du plan complexe.

Le Théorème 3 décrit la distribution des zéros de $I_{n}\left(R_{n} z ; \lambda, E_{1 / \rho}\right)$ dans le voisinage du point $z=1$ et est démontré dans [5] (Théorème 1, p. 10) dans le cas $\lambda=0$. Les résultats correspondant pour $I_{n}\left(n z ; 0, \mathrm{e}^{z}\right)=s_{n}\left(n z, \mathrm{e}^{z}\right)$ et $I_{n}\left(n z ; 1, \mathrm{e}^{z}\right)=-t_{n+1}\left(n z, \mathrm{e}^{z}\right)$ sont obtenus dans [9] et [11]. Le Théorème 4 peut être consideré comme une generalisation des Théorèmes 2 and 3 de [5]. Ce qui est surprenant, c'est que si on prolonge la courbe $S_{1}(\rho)$ à l'exterieur du disque unité par la courbe $S_{2}(\rho)$ qui a la même équation dans les coordonées polaires que $S^{\prime}(\rho)$, le comportement de $I_{n}\left(R_{n} z ; \lambda, E_{1 / \rho}\right)$ dans un voisinage de $S^{\prime}(\rho)$ et $S_{2}(\rho)$ est aussi similair. 
Pour étudier le comportement asymptotique de $I_{n}\left(R_{n} z ; \lambda, E_{1 / \rho}\right)$, on l'écrit sous la forme $I_{n}\left(R_{n} z ; \lambda\right.$, $\left.E_{1 / \rho}\right)=(1-\lambda) E_{1 / \rho}\left(R_{n} z\right)-t_{n+1}\left(R_{n} z, E_{1 / \rho}\right)$. Le comportement asymptotique du premier terme est bien connu. Pour $|z|<1$, le comportement asymptotique du second terme a été étudié dans [5]. Pour $|z|>1$, on utilise la représentation $t_{n+1}\left(R_{n} z, E_{1 / \rho}\right)=\left(R_{n} z\right)^{n+1} E_{1 / \rho}\left(R_{n} z, 1+\frac{n+1}{\rho}\right)$, où $E_{1 / \rho}(z, \mu)$ est la fonction généralisée de Mittag-Leffler définie (3.4). Cette fonction $E_{1 / \rho}(z, \mu)$ admet une représentation integrale commode ([4], p. 127). En appliquant la méthode de Laplace pour l'integral correspondant, on détermine le comportement asymptotique (quand $n \rightarrow \infty)$ de $t_{n+1}\left(R_{n} z, E_{1 / \rho}\right)$ à l'exterieur du disque unité.

\section{Introduction}

For a transcendental entire function

denote by

$$
f(z)=\sum_{k=0}^{\infty} a_{k} z^{k}, \quad a_{0}>0,
$$

$$
s_{n}(z, f)=\sum_{k=0}^{n} a_{k} z^{k} \quad \text { and } \quad t_{n}(z, f)=\sum_{k=n}^{\infty} a_{k} z^{k}
$$

its $n$th section and $n$th tail respectively. Denote by $R_{1}, R_{2}, R_{3}, \ldots$ the discontinuity points of the central index of $f$. One has $\lim _{n \rightarrow \infty} R_{n}=\infty$ (see [8, pp. 5-6]). Let $\mathcal{M}_{n}(\lambda, f), \lambda \in \mathbb{C}$, be the set of all roots of the equation $I_{n}\left(R_{n} z ; \lambda, f\right)=0$, where

$$
I_{n}\left(R_{n} z ; \lambda, f\right)=(1-\lambda) s_{n}\left(R_{n} z, f\right)-\lambda t_{n+1}\left(R_{n} z, f\right) .
$$

In particular, $\mathcal{M}_{n}(0, f)$ coincides with the set of zeros of $s_{n}\left(R_{n} z, f\right)$ and $\mathcal{M}_{n-1}(1, f)$ coincides with the set of zeros of $t_{n}\left(R_{n-1} z, f\right)$. Define $\mathcal{M}(\lambda, f)$ to be the set of all accumulation points of $\bigcup_{n=1}^{\infty} \mathcal{M}_{n}(\lambda, f)$.

In 1924, Szegö [10] proved a remarkable theorem on the asymptotic behavior of the roots of the equation $I_{n}\left(n z ; \lambda, \mathrm{e}^{z}\right)=0$ (note that $R_{n}=n$ for $\left.f(z)=\mathrm{e}^{z}\right)$, wherein the so-called Szegö curve $S:=\left\{z:\left|z \mathrm{e}^{1-z}\right|=1\right\}$ played a key role.

Theorem S ([10]). - One has (i) $\mathcal{M}\left(0, \mathrm{e}^{z}\right)=S \cap\{z:|z| \leqslant 1\}$, (ii) $\mathcal{M}\left(1, \mathrm{e}^{z}\right)=S \cap\{z:|z| \geqslant 1\}$, (iii) $\mathcal{M}\left(\lambda, \mathrm{e}^{z}\right)=S$ for $\lambda \neq 0,1$.

In [5], Edrei, Saff and Varga studied the distribution of the zeros of sections $s_{n}\left(R_{n} z, E_{1 / \rho}\right)$ of the MittagLeffler function of order $\rho>1$,

$$
E_{1 / \rho}(z)=\sum_{j=0}^{\infty} \frac{z^{j}}{\Gamma(1+j / \rho)}, \quad 1<\rho<\infty .
$$

For the function $E_{1 / \rho}(z)$ (see [7, p. 26]), we have $R_{n}=\Gamma(1+n / \rho) / \Gamma(1+(n-1) / \rho)$. Consider the main result of [5]. Edrei, Saff and Varga [5] discovered that the zeros of $s_{n}\left(R_{n} z, E_{1 / \rho}\right)$ are related with the curve $S_{1}(\rho)=S^{\prime}(\rho) \cup S^{\prime \prime}(\rho)$, where

$$
\begin{aligned}
& S^{\prime}(\rho)=\left\{z=r \mathrm{e}^{\mathrm{i} \phi}: r \leqslant 1,|\phi| \leqslant \frac{\pi}{2 \rho}, r^{\rho} \cos (\phi \rho)-1-\rho \log r=0\right\}, \\
& S^{\prime \prime}(\rho)=\left\{z=r \mathrm{e}^{\mathrm{i} \phi}: \frac{\pi}{2 \rho}<\phi<2 \pi-\frac{\pi}{2 \rho}, r=\mathrm{e}^{-1 / \rho}\right\} .
\end{aligned}
$$

The arguments of the zeros of $E_{1 / \rho}(z)$ for $1<\rho<\infty$ tend to $\pm \pi /(2 \rho)$ as $|z| \rightarrow \infty$. Hence, there are zeros of $s_{n}\left(R_{n} z, E_{1 / \rho}\right)$ whose arguments are close to $\pm \pi /(2 \rho)$. Denote

$$
\mathcal{M}_{0}\left(\lambda, E_{1 / \rho}\right)=\mathcal{M}\left(\lambda, E_{1 / \rho}\right) \backslash\left\{z: \arg z= \pm \frac{\pi}{2 \rho}\right\} .
$$

Edrei, Saff and Varga proved the following theorem which is an analogue of part (i) of Theorem S. 


\section{TheOREM ESV $([5]) .-\mathcal{M}_{0}\left(0, E_{1 / \rho}\right)=S_{1}(\rho)$.}

This theorem is a corollary of much more precise and complicated results of [5] related to the description of zero-free regions for $s_{n}\left(R_{n} z, E_{1 / \rho}\right)$. The similarity between the zero distribution of sections of $E_{1 / \rho}(z)$ (given by Theorem ESV) and the zero distribution of sections of $\mathrm{e}^{z}$ (given by Theorem S, part (i)) provokes the following questions. Does an analogue of Szegö's result hold for $I_{n}\left(R_{n} z ; \lambda, E_{1 / \rho}\right)$ ? What is the analogue of the part of Szegö's curve $S$ lying in the exterior of the unit disc? What is the description of zero-free regions for $I_{n}\left(R_{n} z ; \lambda, E_{1 / \rho}\right)$ for arbitrary $\lambda \in \mathbb{C}$ ? Can analogues of other results of [5] related to asymptotic properties of $s_{n}\left(R_{n} z, E_{1 / \rho}\right)=I_{n}\left(R_{n} z ; 0, E_{1 / \rho}\right)$ be developed for $I_{n}\left(R_{n} z ; \lambda, E_{1 / \rho}\right)$ ? The aim of our work is to answer these questions.

\section{Results}

Denote by

$$
S(\rho)=S_{1}(\rho) \cup S_{2}(\rho), \quad \rho>1,
$$

where

$$
S_{2}(\rho)=\left\{z=r \mathrm{e}^{\mathrm{i} \phi}: r \geqslant 1,|\phi| \leqslant \frac{\pi}{2 \rho}, r^{\rho} \cos (\rho \phi)-1-\rho \log r=0\right\} .
$$

The first result of our work can be considered as a complete analogue of Szegö's Theorem $S$.

Theorem 1. - One has (i) $\mathcal{M}_{0}\left(0, E_{1 / \rho}\right)=S_{1}(\rho)$, (ii) $\mathcal{M}_{0}\left(1, E_{1 / \rho}\right)=S_{2}(\rho)$, (iii) $\mathcal{M}_{0}\left(\lambda, E_{1 / \rho}\right)=$ $S(\rho)$, for $\lambda \neq 0,1$.

Theorem 1 answers the question how to continue the curve $S(\rho)$ into the exterior of the unit disc. It also implies that zeros of $I_{n}\left(R_{n} z ; \lambda, E_{1 / \rho}\right)$ may lie only in the vicinity of curve $S(\rho)$ and two rays $\arg z= \pm \pi /(2 \rho)$. Note that the curve $S(\rho)$ has asymptotes $\arg z= \pm \pi /(2 \rho)$, while the original Szegö curve $S$ does not have any linear asymptote.

For given $\delta_{1}>0, \delta_{2}>0$ and $h>0$, let us introduce the following regions.

$$
\begin{aligned}
& \Omega_{1}=\left\{z=r \mathrm{e}^{\mathrm{i} \phi}: \delta_{1}<r \leqslant 1,|z-1| \geqslant \delta_{1},|\phi| \leqslant \frac{\pi}{2 \rho}-\delta_{2}, r^{\rho} \cos (\rho \phi)-1-\rho \log r \geqslant 0\right\}, \\
& \Omega_{2}=\left\{z=r \mathrm{e}^{\mathrm{i} \phi}:|\phi| \leqslant \frac{\pi}{2 \rho}-\delta_{2}, r^{\rho} \cos (\rho \phi)-1-\rho \log r \leqslant-h\right\}, \\
& \Omega_{3}=\left\{z=r \mathrm{e}^{\mathrm{i} \phi}: r \geqslant \mathrm{e}^{-1 / \rho}+h,|\phi| \geqslant \frac{\pi}{2 \rho}+\delta_{2}\right\}, \\
& \Omega_{4}=\left\{z=r \mathrm{e}^{\mathrm{i} \phi}: \delta_{1}<r \leqslant \mathrm{e}^{-1 / \rho}-h,|\phi| \geqslant \frac{\pi}{2 \rho}+\delta_{2}\right\}, \\
& \Omega_{5}=\left\{z=r \mathrm{e}^{\mathrm{i} \phi}: r \geqslant 1,|\phi| \leqslant \frac{\pi}{2 \rho}-\delta_{2}, r^{\rho} \cos (\rho \phi)-1-\rho \log r \geqslant h\right\} .
\end{aligned}
$$

The next theorem deals with the zero-free regions of $I_{n}\left(R_{n} z ; \lambda, E_{1 / \rho}\right)$.

THEOREM 2.-Let $\delta_{1}, \delta_{2}$ and $h$ be given positive constants. Then, for all sufficiently large $n$, $I_{n}\left(R_{n} z ; \lambda, E_{1 / \rho}\right)$ has no zeros in $\bigcup_{i=1}^{5} \Omega_{i}$.

Theorem 2 can be viewed as an extension of Theorem 5 of [5].

The next two theorems give information on the zero distribution of $I_{n}\left(R_{n} z ; \lambda, E_{1 / \rho}\right)$ in the neighborhood of points on the curve $S(\rho)$. The distribution in the neighborhood of the point $z=1$ is characterized by the use of the complementary error function,

$$
\operatorname{erfc}(\zeta)=1-\frac{2}{\pi} \int_{0}^{\zeta} \mathrm{e}^{-v^{2}} \mathrm{~d} v
$$

THEOREM 3. - As $n \rightarrow \infty$, we have

$$
\left(1+\left(\frac{2}{\rho n}\right)^{1 / 2} \zeta\right)^{-n}\left\{E_{1 / \rho}\left(R_{n}\right)\right\}^{-1} I_{n}\left(R_{n}\left(1+\left(\frac{2}{\rho n}\right)^{1 / 2} \zeta\right) ; \lambda, E_{1 / \rho}\right) \rightarrow \mathrm{e}^{\zeta^{2}}\left\{\frac{\operatorname{erfc}(\zeta)}{2}-\lambda\right\}
$$


uniformly on every compact set of the $\zeta$-plane.

Theorem 3 can be viewed as an extension of Theorem 1 of [5]. The proof of Theorem 3 is based on the well-known asymptotic expression for Mittag-Leffler function of order $\rho>1$ and Theorem 1 from [5].

THEOREM 4. - I. Let $\xi=\xi(\phi), 0<\phi<\frac{\pi}{2 \rho}$, be a fixed point on $S^{\prime}(\rho) \cup S_{2}(\rho)$. Let $\tau=|\zeta|^{\lambda} \sin (\phi \rho)-\rho \phi$, and let the sequences $\left\{\tau_{n}\right\}_{n=1}^{\infty}$ and $\left\{\varepsilon_{n}\right\}_{n=1}^{\infty}$ be defined by the conditions

Then, as $n \rightarrow \infty$,

$$
\tau_{n} \equiv \frac{\tau}{\rho} n(\bmod 2 \pi), \quad-\pi<\tau_{n} \leqslant \pi, \quad \text { and } \quad \varepsilon_{n}=\frac{\log n}{2\left(1-\xi^{\rho}\right) n}-\frac{\zeta-\mathrm{i} \tau_{n}}{\left(1-\xi^{\rho}\right) n} .
$$

$$
\frac{I_{n}\left(R_{n} \xi\left(1+\varepsilon_{n}\right) ; \lambda, E_{1 / \rho}\right) \Gamma(1+n / \rho)}{R_{n}^{n} \xi^{n}\left(1+\varepsilon_{n}\right)^{n}} \rightarrow \begin{cases}(1-\lambda)(2 \pi \rho)^{1 / 2} \mathrm{e}^{(\rho+1) /(2 \rho)\left(\xi^{\rho}-1\right)} \mathrm{e}^{\zeta}-\frac{\xi}{1-\xi} & \text { if }|\xi|<1, \\ -\lambda(2 \pi \rho)^{1 / 2} \mathrm{e}^{(\rho+1) /(2 \rho)\left(\xi^{\rho}-1\right)} \mathrm{e}^{\zeta}-\frac{\xi}{1-\xi} & \text { if }|\xi|>1,\end{cases}
$$

uniformly on every compact set of the $\zeta$-plane.

II. Let $\xi=\mathrm{e}^{-1 / \rho} \mathrm{e}^{\mathrm{i} \phi}, \frac{\pi}{2 \rho}<\phi \leqslant \pi$, be a fixed point on $S^{\prime \prime}(\rho)$, and let the sequences $\left\{\tau_{n}^{\prime}\right\}_{n=1}^{\infty}$ and $\left\{\varepsilon_{n}^{\prime}\right\}_{n=1}^{\infty}$ be defined by the conditions

Then

$$
\tau_{n}^{\prime} \equiv(n+1) \phi(\bmod 2 \pi), \quad-\pi<\tau_{n}^{\prime} \leqslant \pi, \quad \text { and } \quad \varepsilon_{n}^{\prime}=\left(\frac{1}{2}-\frac{1}{\rho}\right) \frac{\log n}{n}-\frac{\zeta-\mathrm{i} \tau_{n}^{\prime}}{n+1} .
$$

$$
I_{n}\left(R_{n} \xi\left(1+\varepsilon_{n}^{\prime}\right) ; \lambda, E_{1 / \rho}\right) \frac{\Gamma(1+n / \rho)}{R_{n}^{n} \xi^{n}\left(1+\varepsilon_{n}^{\prime}\right)^{n}} \rightarrow \frac{(\lambda-1)\left(2 \pi \mathrm{e}^{(1-\rho) / \rho}\right)^{1 / 2}}{\rho^{1 / 2-1 / \rho} \Gamma(1-1 / \rho)} \mathrm{e}^{-\zeta}-\frac{\xi}{1-\xi}
$$

uniformly on every compact set of the $\zeta$-plane.

It is worth mentioning that the arguments of $I_{n}$ in Theorem 4 are the same as in Theorems 2 and 3 of [5].

\section{Method of proof}

The basis of our study is the following theorem that deals with the asymptotic expressions for $I_{n}\left(R_{n} z ; \lambda, E_{1 / \rho}\right)$ in different domains of $\mathbb{C}$.

THEOREM A. - Let $\delta_{1}, \delta_{2}$ be given positive constants, and $\rho>1$. Then, as $n \rightarrow \infty$,

$$
\begin{aligned}
& \frac{I_{n}\left(R_{n} z ; \lambda, E_{1 / \rho}\right) \Gamma(1+n / \rho)}{R_{n}^{n} z^{n}}=-\lambda \rho \frac{\mathrm{e}^{R_{n}^{\rho} z^{\rho}} \Gamma(1+n / \rho)}{R_{n}^{n} z^{n}}(1+\mathrm{o}(1))-\frac{z}{1-z}(1+\mathrm{o}(1)), \\
& \text { if } z \in\left\{z=r \mathrm{e}^{\mathrm{i} \phi}: r \geqslant 1,|\phi| \leqslant \frac{\pi}{2 \rho},|z-1| \geqslant \delta_{1}\right\} \\
& \frac{I_{n}\left(R_{n} z ; \lambda, E_{1 / \rho}\right) \Gamma(1+n / \rho)}{R_{n}^{n} z^{n}}=(1-\lambda) \rho \frac{\mathrm{e}^{R_{n}^{\rho} z^{\rho}} \Gamma(1+n / \rho)}{R_{n}^{n} z^{n}}(1+\mathrm{o}(1))-\frac{z}{1-z}(1+\mathrm{o}(1)), \\
& \quad \text { if } \quad z \in\left\{z=r \mathrm{e}^{\mathrm{i} \phi}: \delta_{1}<r \leqslant 1,|\phi| \leqslant \frac{\pi}{2 \rho},|z-1| \geqslant \delta_{1}\right\}, \\
& \frac{I_{n}\left(R_{n} z ; \lambda, E_{1 / \rho}\right) \Gamma(1+n / \rho)}{R_{n}^{n} z^{n}}=\frac{(\lambda-1)}{\Gamma(1-1 / \rho)} \frac{\Gamma(1+n / \rho)}{R_{n}^{n+1} z^{n+1}}(1+\mathrm{o}(1))-\frac{z}{1-z}(1+\mathrm{o}(1)), \\
& \text { if } \quad z \in\left\{z=r \mathrm{e}^{\mathrm{i} \phi}: r>\delta_{1},|\phi| \geqslant \frac{\pi}{2 \rho}+\delta_{2}\right\}
\end{aligned}
$$

In all expressions above, $\mathrm{o}(1)$ is uniform with respect to $z$.

We remark that, in the special case $\lambda=1,|z|<1$, one can find the asymptotic expression for $I_{n}\left(R_{n} z ; 1, E_{1 / \rho}\right)$ in [5, Lemma 9.2]. 
Theorems 1, 2 and 4 can be derived from Theorem A. The proof of Theorem A consists of three steps. Step 1 . We rewrite $I_{n}\left(R_{n} z ; \lambda, E_{1 / \rho}\right)$ as follows

$$
\begin{aligned}
I_{n}\left(R_{n} z ; \lambda, E_{1 / \rho}\right) & =(1-\lambda) E_{1 / \rho}\left(R_{n} z\right)-t_{n+1}\left(R_{n} z, E_{1 / \rho}\right) \\
& =(1-\lambda) E_{1 / \rho}\left(R_{n} z\right)-\left(R_{n} z\right)^{n+1} E_{1 / \rho}\left(R_{n} z ; 1+\frac{n+1}{\rho}\right),
\end{aligned}
$$

where

$$
E_{1 / \rho}(z, \mu)=\sum_{k=0}^{\infty}\left\{\Gamma\left(\mu+\frac{k}{\rho}\right)\right\}^{-1} z^{k}, \quad \rho>0, \mu \in \mathbb{C},
$$

is a generalized Mittag-Leffler function studied by Djrbashian in [4, p. 117].

Step 2. Using a well-known asymptotic expression for $E_{1 / \rho}\left(R_{n} z\right)$ and an integral representation (see [4]) for $E_{1 / \rho}\left(R_{n} z, 1+(n+1) / \rho\right)$, we get the following expressions for $I_{n}\left(R_{n} z, \lambda, E_{1 / \rho}\right)$ :

$$
\begin{aligned}
& I_{n}\left(R_{n} z ; \lambda, E_{1 / \rho}\right)=-\lambda \rho \mathrm{e}^{R_{n}^{\rho} z^{\rho}}(1+\mathrm{o}(1))-\frac{\rho\left(R_{n} z\right)^{n+1}}{2 \pi \mathrm{i}} \int_{L\left(\pi /(2 \rho)+\delta_{2} / 2, R_{n}\right)} \frac{\mathrm{e}^{\zeta^{\rho}} \zeta^{-(n+1)}}{\zeta-R_{n} z} \mathrm{~d} \zeta, \\
& \quad \text { if }|z|>1 \text { and }|\arg z| \leqslant \pi /(2 \rho), \\
& I_{n}\left(R_{n} z ; \lambda, E_{1 / \rho}\right)=\frac{(\lambda-1)}{R_{n} z \Gamma(1-1 / \rho)}(1+\mathrm{o}(1))-\frac{\rho\left(R_{n} z\right)^{n+1}}{2 \pi \mathrm{i}} \int_{L\left(\pi /(2 \rho)+\delta_{2} / 2, R_{n}\right)} \frac{\mathrm{e}^{\zeta^{\rho}} \zeta^{-(n+1)}}{\zeta-R_{n} z} \mathrm{~d} \zeta, \\
& \quad \text { if }|z|>0 \text { and }|\arg z|>\pi /(2 \rho)+\delta_{2} /(2 \rho), \\
& I_{n}\left(R_{n} z ; \lambda, E_{1 / \rho}\right)=(1-\lambda) \rho \mathrm{e}^{R_{n}^{\rho} z^{\rho}}(1+\mathrm{o}(1))-\frac{\rho\left(R_{n} z\right)^{n+1}}{2 \pi \mathrm{i}} \int_{L\left(\pi /(2 \rho)+\delta_{2} / 2, R_{n}\right)} \frac{\mathrm{e}^{\zeta^{\rho}} \zeta^{-(n+1)}}{\zeta-R_{n} z} \mathrm{~d} \zeta, \\
& \quad \text { if } 0<|z|<1 \text { and }|\arg z| \leqslant \pi /(2 \rho),
\end{aligned}
$$

where $L(\alpha, H)(H>0,0<\alpha \leqslant \pi)$ is a contour following nondecreasing direction of $\arg \zeta$ and consisting of two rays $\{\arg \zeta= \pm \alpha,|\zeta| \geqslant H\}$ and an $\operatorname{arc}\{-\alpha \leqslant \arg \zeta \leqslant \alpha\}$ of a circle $|\zeta|=H$.

Step 3 . We show that the main contribution to

$$
K_{n}(z):=\int_{L\left(\pi /(2 \rho)+\delta_{2} / 2, R_{n}\right)} \frac{\mathrm{e}^{\zeta^{\rho}} \zeta^{-(n+1)}}{\zeta-R_{n} z} \mathrm{~d} \zeta
$$

comes from the neighborhood of the point $\zeta=R_{n}$, and we find an asymptotic expression for $K_{n}(z)$ by using Laplace's method.

Acknowledgements. The author is grateful to Professors I.V. Ostrovskii and C.Y. Ylldırım for constant attention to this work and for useful discussions.

\section{References}

[1] J.D. Buckholtz, A characteriation of the exponential series, Part II, Amer. Math. Monthly 73 (1966) 121-123.

[2] A.J. Carpenter, R.S. Varga, J. Waldvogel, Asymptotics for the partial sums of e ${ }^{z}$, I, Rocky Mountain J. Math. 21 (1) (1991) 99-120.

[3] J. Dieudonné, Sur les zéros des polynomes-sections de e e ${ }^{x}$, Bull Soc. Math. France 70 (1935) 333-351.

[4] M.M. Djrbashian, Integral Transforms and Representations of Functions in the Complex Domain, Nauka, Moskow, 1966 (in Russian).

[5] A. Edrei, E.B. Saff, R.S. Varga, Zeros of sections of power series, in: Lecture Notes in Math., Vol. 1002, 1983, pp. $1-115$.

[6] I.V. Ostrovskii, On zero distribution of sections and tails of power series, Israel Math. Conf. Proc. 15 (2001) 297-310.

[7] G. Pólya, G. Szegö, Problems and Theorems in Analysis, I, Springer-Verlag, Berlin, 1976.

[8] G. Pólya, G. Szegö, Problems and Theorems in Analysis, II, Springer-Verlag, Berlin, 1976.

[9] D.J. Newman, T.J. Rivlin, The zeros of the partial sums of the exponential function, J. Approx. Theory 5 (1972) 405-412.

[10] G. Szegö, Über eine Eigenschaft der Exponentialreihe, Sitzungsber. Berliner Math. Gesellsch. 23 (1924) 50-64.

[11] C.Y. Yildırım, A sum over the zeros of partial sums of e ${ }^{z}$, J. Ramanujan Math. Soc. $6(1,2)(1991)$ 51-66. 